\title{
Predicted colours for simple stellar populations
}

\section{The case of old stellar clusters}

\author{
E. Brocato ${ }^{1,2}$, V. Castellani ${ }^{3,4}$, F.M. Poli ${ }^{5}$, and G. Raimondo ${ }^{1,2}$ \\ 1 Osservatorio Astronomico di Collurania, Via M. Maggini, I-64100 Teramo, Italy \\ e-mail: brocato, raimondo@astrte.te.astro.it \\ ${ }^{2}$ Istituto Nazionale di Fisica Nucleare, LNGS, L'Aquila, Italy \\ 3 Dipartimento di Fisica, Università di Pisa, I-56100 Pisa, Italy \\ e-mail: vittorio@astr18pi.difi.unipi.it \\ 4 Istituto Nazionale di Fisica Nucleare, I-56100 Pisa, Italy \\ 5 Associazione Euratrom-ENEA CRE, I-00044 Frascati, Italy \\ e-mail: fpoli@frascati.enea.it
}

Received February 10; accepted July 3, 2000

\begin{abstract}
This paper presents theoretical integrated colours of old stellar populations as computed adopting an homogeneous set of stellar models, covering all the major evolutionary phases of globular cluster stars. We show that adopting Reimers parameterization of mass loss rates, the choice $\eta=0.4$ gives synthetic CM diagrams of simple stellar populations in agreement with the typical dependence of globular cluster CM diagrams on metallicity. We present theoretical $U-B, B-V$, $V-R$ and $V-I$ integrated colours for cluster metallicity in the range $Z=0.0001$ to 0.02 and for age between 8 and 15 Gyr. The stochastic occurrence of luminous post-AGB stars is briefly discussed. We find that for an age $t \sim 15$ Gyr the predicted integrated colours appear in good agreement with available data for both galactic and LMC old globulars. We discuss the uncertainties of integrated colours due to statistical fluctuations in the number of luminous stars, giving for each colour the expected uncertainty as a function of the cluster integrated $V$-magnitude. Comparison with Kurth et al. (1999) discloses that the still existing differences in the evolutionary results appear of minor relevance as far as integrated cluster colours is concerned. Finally, we show that reasonable uncertainties either in the cluster age or in the efficiency of mass loss have marginal effects on the predicted colours.
\end{abstract}

Key words: galaxy: globular clusters: general galaxies: star clusters — galaxies: Magellanic Clouds stars: AGB and post-AGB — Stars: Hertzsprung-Russel (HR) and $\mathrm{C}-\mathrm{M}$ diagrams

Send offprint requests to: E. Brocato

\section{Introduction}

In spite of the tremendous improvement of the new generation telescopes (VLT, HST, KECK etc.), severe physical limits do not allow to resolve distant stellar systems, and integrated colours and spectra will remain for a long time the only way to investigate the evolutionary history of stellar systems beyond the Local Group of galaxies. In this context, theoretical predictions concerning simple stellar populations (SSP), i.e. stellar populations with a common age and chemical composition, appear of paramount relevance, since SSP represent the fundamental ingredient to approach the observational evidences from more complex stellar systems.

In the recent past several authors have presented SSP models (see, e.g., Buzzoni 1989; Leitherer et al. 1996; Tantalo et al. 1996 (TCBF); Vazdekis et al. 1996 (VCPB); Maraston 1998; Kurth et al. 1999 (KFF)) for a wide range of ages and metallicities, already providing valuable scenarios on this matter. However, only Covino et al. (1994) make a brief discussion on the effect of different assumptions on the mass loss rate, whereas one knows that the HB morphology is critically affected by the amount of mass lost during the RGB phase (see, e.g., Iben \& Renzini 1983; Jørgensen \& Thejll 1993). Moreover, we still lack a detailed evaluation of the influence of statistical fluctuations on the cluster integrated colours, a problem which has been addressed only by Tantalo et al. (1996).

In a previous paper (Brocato et al. 1999a, Paper I) we have recently presented theoretical integrated colours of simple stellar populations with ages smaller or of the order of 5 Gyr, testing the results on a suitable sample of Large Magellanic Cloud stellar clusters. As discussed in that 
paper, the given upper limit to cluster ages was suggested by the opportunity to minimize uncertainties about mass loss which, in old stellar clusters, governs the temperature of horizontal branch (HB) stars, affecting the predicted colours. This paper will now cross this limit, exploring the sensitivity of the theoretical scenario for old stellar clusters to reasonable assumptions about the efficiency of mass loss.

Following a slightly unconventional approach, we will start our investigation by testing first the capability of the adopted evolutionary models to reproduce the CM diagram distribution of stars in selected globular clusters. This appears to us as a preliminary but relevant step, aiming to ground the investigation on reasonably firm basis. Such a test will be discussed in Sect. 3, after presenting in Sect. 2 preliminary information on the adopted evolutionary scenario and on the computational procedure. In Sect. 4 we will make use of integrated colours for a template population to investigate the effects of statistical fluctuations. According to the procedure already envisaged in Paper I, we will correlate the size of predicted fluctuations to the absolute integrated $V$ magnitude of the clusters, allowing a quantitative estimate of the not negligible uncertainty affecting a large portion of galactic globular clusters. We will finally present theoretically predicted colours as a function of age, metallicity and/or efficiency of mass loss, making suitable comparisons with observations and with previous available results. A short discussion will close the paper.

\section{Computational procedures}

As well known, theoretical predictions of cluster colours make use of suitable libraries of evolutionary tracks to produce synthetic stellar clusters, i.e., to simulate the $\mathrm{CM}$ distribution of stars for each given assumption about the cluster age and original chemical composition. In such a procedure, one is usually neglecting faint evolutionary phases which cannot sensitively contribute to the total cluster luminosity. However, since synthetic stellar clusters are of relevance in a much more general context than in predicting colours, the evolutionary library adopted in Paper I has been implemented to cover with suitable theoretical predictions all the evolutionary phases of cluster stars, from the Very Low Mass MS stars, at the lower mass limit for $\mathrm{H}$ burning ignition, to the final sequence of cooling White Dwarfs. As a result, with the present libraries the program can produce "complete" synthetic clusters in the range of ages $50 \mathrm{Myr}$ to $20 \mathrm{Gyr}$ and for metallicity from $Z=0.0001$ to $Z=0.02$. Figure 1 gives an example of these predictions, as given for a synthetic cluster with the given values of age and chemical composition.

In the present form, the bulk of the code relies on the set of homogeneous evolutionary computations presented by Straniero \& Chieffi (1991), Castellani et al. (1991),

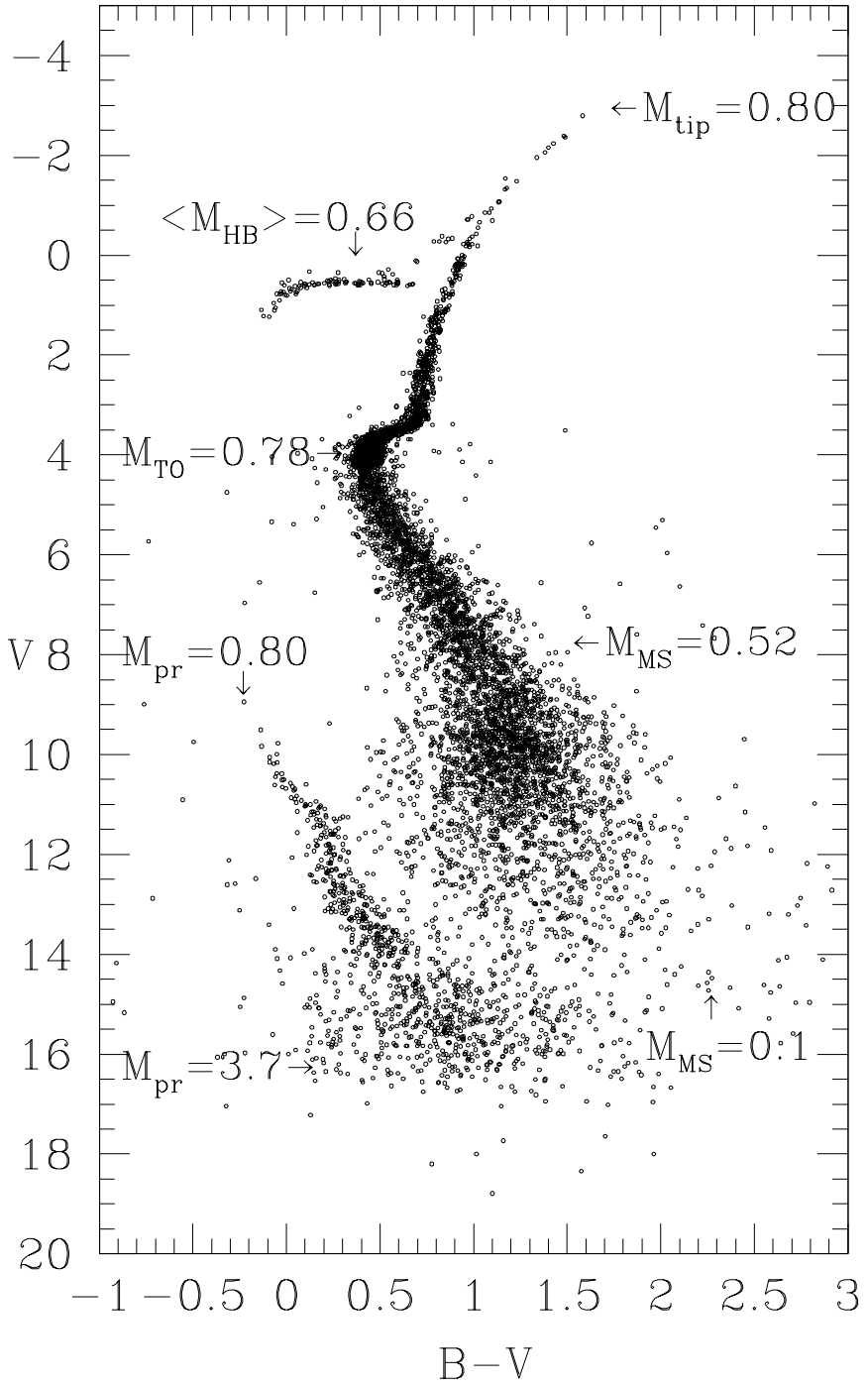

Fig. 1. The predicted CM diagram location of stars in a cluster with age $t=15 \mathrm{Gyr}$ and $Y=0.23, Z=0.001$, with a suitable simulation of photometric uncertainties. $M_{\mathrm{MS}}$ is the mass of Main Sequence star at the corresponding luminosity level; $M_{\mathrm{TO}}$ is the mass of the star at the Turn Off point; $M_{\mathrm{tip}}$ is the mass at the RGB tip; $\left\langle M_{\mathrm{HB}}>\right.$ gives the actual mean value of HB stars after mass loss, whereas the two labelled $M_{\mathrm{pr}}$ values give the progenitor mass (i.e. the mass in MS) of the stars along the WD sequence

Castellani et al. (1992a), Cassisi et al. (1994), covering both the $\mathrm{H}$ and the He burning phase for stars with original masses in the range $0.6-9.0 M_{\odot}$ and where the He burning phase is followed till the Carbon ignition or, alternatively, the onset of the thermal pulse phase. In the last case, the relevant one for old globulars, the tracks have been prolonged through the thermal pulse phase according to the semianalitical procedure envisaged by Groenewegen \& De Jong (1993, see also Marigo et al. 1996). The above evolutionary computations have been implemented with theoretical predictions about VLM structures from Cassisi et al. (2000) whereas 


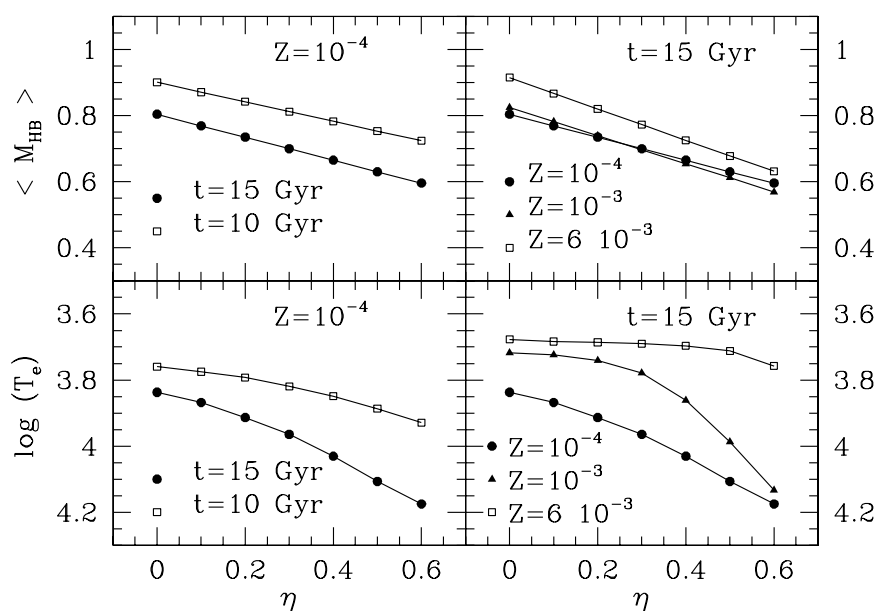

Fig. 2. Predicted masses and temperatures of HB stars as a function of $\eta$ for the labelled assumptions about the cluster age or metallicity

cooling White Dwarf sequences have been finally evaluated according to the Brocato et al. (1999b) procedure, as based on evolutionary data by Wood (1992).

A detailed description of the program can be found in Poli (1997) and Paper I. Here we notice that mass loss has been taken into account by adopting Reimers formula

$\dot{M}=-410^{-13} \eta \frac{L}{g R} \frac{M_{\odot}}{\mathrm{yr}}$

connecting the amount of mass loss to the stellar parameters $L, M$ and $T_{\mathrm{e}}$, with $\eta$ as a free parameter governing the efficiency of the process. Since the evolution of a Red Giant structure is largely independent of the amount of mass loss (see Castellani \& Castellani 1993) the above relation can be integrated over the time all along the $\mathrm{H}$ burning evolution of models without mass loss in order to obtain the total amount of mass lost during this phase and, in turn, the mass of the new born He burning HB star.

To orientate the reader in such a scenario, Fig. 2 gives the predicted mass and effective temperature of new born HB stars as a function of $\eta$ for selected assumption about the cluster age or metallicity. As already known, one recognizes that decreasing the HB mass (i.e., increasing $\eta$ and/or the age) HB stars become hotter (and fainter). One should also notice the critical dependence of the HB temperature of $Z=10^{-3}$ models on the mass, to be connected with the observational evidence for extended $\mathrm{HB}$ in intermediate metallicity clusters like M 3 or M 5.

For each given age and chemical composition and for each given assumption on $\eta$, we make use of the above procedure to evaluate the original mass of the star at the tip of the red giant branch and the amount of mass lost, deriving from these values, the mass of the HB star, its $\mathrm{CM}$ diagram location and the lifetime in the HB phase. This lifetime is used to derive the number of expected HB stars $\left(N_{\mathrm{HB}}\right)$ through a suitable proportion with the
Table 1. The expected number of P-AGB stars as a function of the total absolute cluster magnitude $M_{V}^{\text {tot }} . N_{\text {cross }}^{1}$ refers to the model with $M_{\mathrm{H}}=0.55 M_{\odot}$ and $N_{\text {cross }}^{2}$ to the model with $M_{\mathrm{H}}=0.525 M_{\odot}$ (Blöcker \& Schönberner 1997)

\begin{tabular}{llll}
\hline$M_{V}^{\text {tot }}$ & $N_{\mathrm{HB}}$ & $N_{\text {cross }}^{1}$ & $N_{\text {cross }}^{2}$ \\
\hline-4.1 & 12 & 0.005 & 0.01 \\
-5.9 & 74 & 0.03 & 0.08 \\
-6.7 & 146 & 0.07 & 0.15 \\
-7.5 & 288 & 0.13 & 0.30 \\
-7.9 & 436 & 0.20 & 0.46 \\
-8.5 & 730 & 0.33 & 0.77 \\
-9.0 & 1166 & 0.53 & 1.22 \\
-10.0 & 2904 & 1.32 & 3.08 \\
-11.0 & 7304 & 3.32 & 7.74 \\
-12.0 & 18326 & 8.33 & 19.45 \\
\hline
\end{tabular}

proper RGB lifetime and the already known number of RGB stars.

As well known, the HB colour distribution observed in the galactic globulars has to be interpreted as an evidence for a dispersion in HB masses, as due to a dispersion in the amount of mass loss. To simulate this occurrence, we follow the prescriptions early given by Rood (1973) by assuming the computed HB mass as the mean value of a stochastic distribution of masses with a given standard deviation, normally in the range $\sigma_{\mathrm{M}}=0.02-0.04$. For each given HB mass, the star is put on its evolutionary track with a random fraction of its He burning lifetime, still evaluating the amount of mass loss (Reimers) during the rising along the AGB phase. As derived by Fig. 4 of Castellani et al. (1992b), if and when the mass of the H-rich envelope decreases below a critical value

$$
\begin{aligned}
M_{\mathrm{cr}}^{\mathrm{env}} & =0.0153 \cdot\left[\log \left(L / L_{\odot}\right)\right]^{2} \\
& -0.0905 \cdot \log \left(L / L_{\odot}+0.1481\right)
\end{aligned}
$$

the star is removed from the AGB and added to the WD sequence. Note that in this way we deliberately neglect the occurrence of luminous stars crossing the HR diagram to reach their final WD structure. Since the typical lifetime of such a crossing is of the order of only $10^{4}-10^{5}$ years, depending on the star mass (Schönberner 1983; Blöcker \& Schönberner 1997), even in rich globulars the occurrence of similar stars is expected as a rare and stochastic phenomenon, as confirmed by observation. Therefore the contribution of these object has to be treated as an additional occurrence. One can easily quantify the expected number ratio of post-AGB to HB stars, as simply given by the ratio of their corresponding evolutionary lifetimes. According to numerical experiments we report in Table 1 the expected P-AGB number $\left(N_{\text {cross }}\right)$ as a function of the cluster integrated absolute magnitude $\left(M_{V}^{\text {tot }}\right)$ together with the corresponding number of the He-burning stars. These results, in general agreement with predictions by Renzini (1998), confirm that for galactic globulars $\left(M_{V}^{\text {tot }}>-10 \mathrm{mag}\right)$ post-AGB stars can only give a stochastic contribution. 


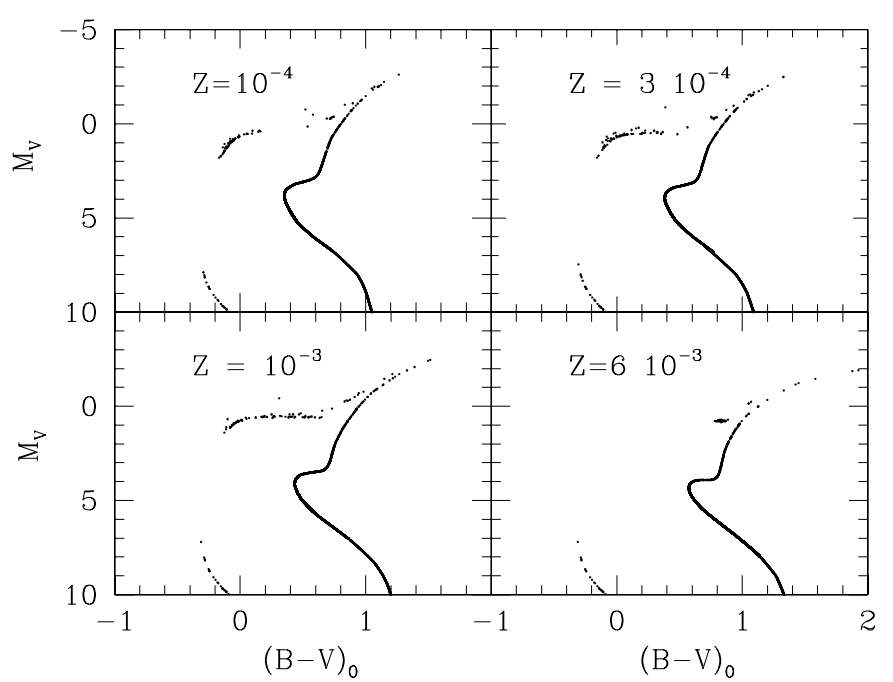

Fig. 3. Predicted CM diagrams for clusters with age $t=15 \mathrm{Gyr}$ and for the labelled assumptions on the metallicity $Z$

Assuming cluster ages in the range $10-15$ Gyr, from preliminary computations we find that the assumption $\eta \sim 0.4$ appears able to nicely reproduce the observed dependence of the HB type on metallicity, when the occurrence of a second parameter is not taken into account. This is shown in Fig. 3, where we report the predicted CM diagram for cluster with an age of 15 Gyr and for the various labelled assumptions about the metallicity, adopting Yale (http://shemesh.gsfc.nasa.gov/iso/color.tblextg) colour temperature relations and bolometric corrections.

\section{The globular cluster test}

As quoted in the introduction of this paper let us verify if and how the adopted theoretical scenario is able to pass the test of actual stellar clusters. To this purpose, we choose the two globulars M 30 (Piotto 2000) and 47 Tuc (Gilliland et al. 1998) representing two extreme metallicities spanned by galactic globulars. The best fitting requires a colour-temperature relation and one finds in the literature several relations which mainly differ in predicting the colour of cool stars (Yale; Lejeune et al. 1997). However, one knows that the theoretical temperature of cool stars is actually a free parameter governed by the assumptions about the mixing length, which can be tuned in such a way that the combination of theoretical temperatures with the adopted colour-temperature relation does reproduce the observed colours of cool stars and, in the present case, the colour of globular cluster Red Giant branches. We found that the combination of the adopted theoretical scenario with Yale transformation naturally account for the CM location of RGB. Such an agreement only means that Yale atmospheres are the most suitable ones to push the theoretical RG branches with our choice about the mixing length in the right place of the $\mathrm{CM}$ diagram: adopting different colour-temperature relations would only require a corresponding variation in the assumed mixing length value, without affecting the final predictions.

Figure 4 shows that adopted theoretical scenario with $\eta=0.4$ is satisfactorily reproducing the CM diagram of both test clusters with reasonable assumptions about the cluster reddenings and distance moduli, i.e., within the currently accepted uncertainties on these values (see, e.g., Reed et al. 1988; Harris 1999; Sandquist et al. 1999).

One may notice that the best fits predict for M 30 and 47 Tuc an age of 14 and 12 Gyr, respectively. As we will discuss in the following, one cannot attribute too much weight to this absolute determination of the ages, because of systematic uncertainties. However, it appears of obvious relevance that a similar difference in ages, with 47 Tuc younger by a couple of Gyr than M 30, has been earlier suggested by Vandenberg et al. (1990) and supported by the recent investigation by Salaris \& Weiss (1998).

As for absolute age determinations, the problem has been largely debated in the current literature and the situation is far from being firmly assessed. Several theoretical investigations have found that new "improved" physic inputs affect stellar models in the direction of decreasing the predicted ages (see for example Chaboyer \& Kim 1995; VandenBerg et al. 1996; Cassisi et al. 1998, 1999). However, in the meantime evidences have been discussed raising serious doubts about the adequacy of such new input physics (Pols et al. 1998; Caputo et al. 1999; Castellani et al. 2000). Taking also into account that the possible (if not probable) efficiency of element sedimentation in globular cluster stars can further reduce the predicted ages (Chaboyer 1995; Castellani et al. 1997; Cassisi et al. 1998) one should regard the ages given by the adopted theoretical scenario as a reasonable upper limit for the cluster ages, real ages being possibly smaller by something of the order of 2 Gyr. However, one could be much more confident in the differential behaviour, which appears scarcely affected by the systematic uncertainties we are dealing with.

\section{Cluster integrated colours}

According to the discussed procedure, we are now in the position of predicting integrated colors for each given assumption about the cluster age and/or chemical composition. As a first step, let us test whether the synthetic cluster procedure is able to reasonably reproduce the integrated colours observed in actual clusters. On very general ground, one expects that synthetic clusters well reproducing the CM diagram distribution of stars should automatically produce reasonable colours. To discuss this point Table 2 compares observed colours for our test clusters, M 30 and 47 Tuc, with the result of theoretical simulations for the selected choices about the cluster age. 

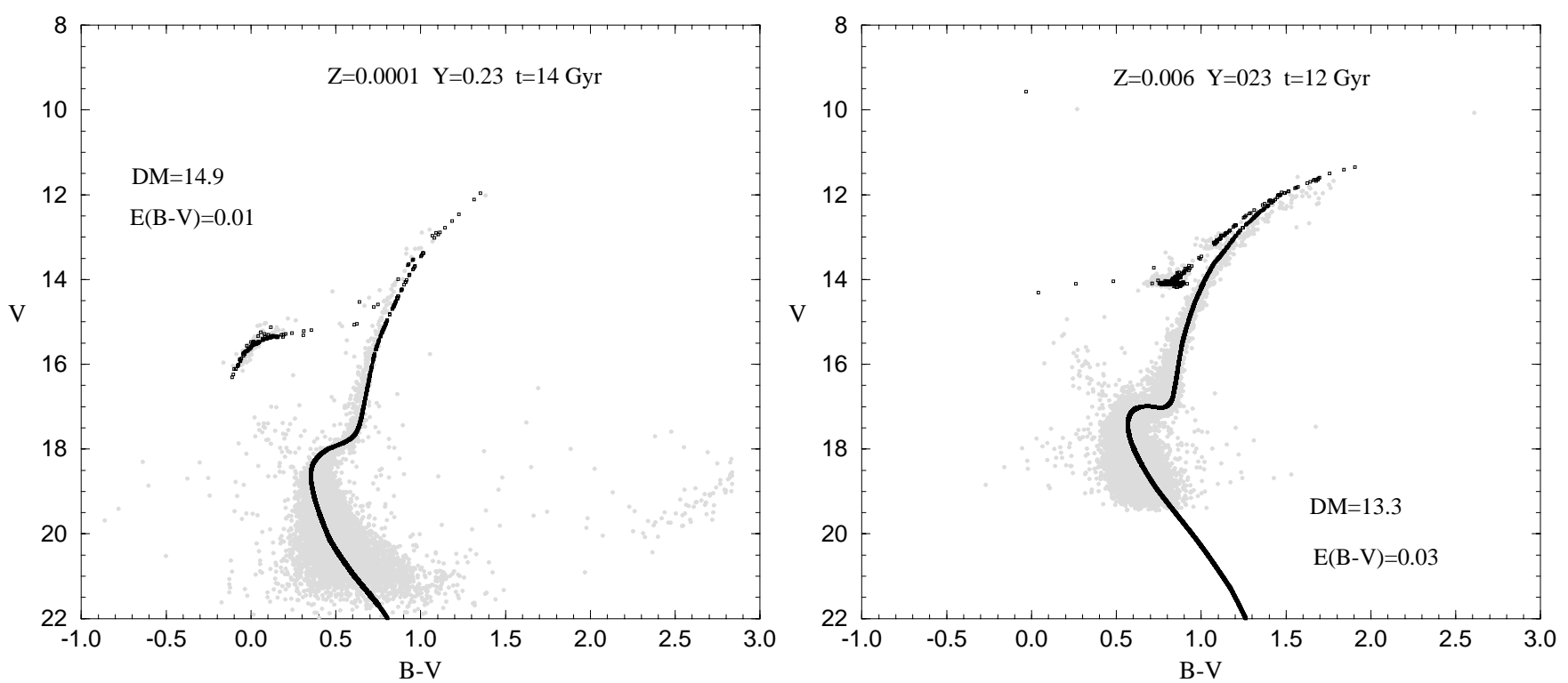

Fig. 4. The best fit of HST CMD of M 30 (left Panel) and 47 Tuc (right panel) with the adopted theoretical isochrones

Table 2. Observed and expected integrated colours for the two prototype globulars M 30 and 47 Tuc. $M_{V}^{0.5}$ is the cluster $V$ absolute magnitude at half-light radius $\left(r^{0.5}\right)$

\begin{tabular}{c|rl|rll|lll|ll}
\hline Cluster & \multicolumn{2}{|c}{$U-B$} & \multicolumn{2}{c}{$B-V$} & \multicolumn{2}{c}{$V-R$} & \multicolumn{2}{c}{$V-I$} \\
\hline M $30\left(M_{V}^{0.5}=-6.6\right)$ & 0.02 & $(.02)$ & 0.59 & $(.04)$ & 0.41 & $(.02)$ & 0.85 & $(.04)$ \\
Synthetic & -0.02 & $(.02)$ & 0.61 & $(.03)$ & 0.43 & $(.02)$ & 0.86 & $(.03)$ \\
47 Tuc $\left(M_{V}^{0.5}=-8.6\right)$ & 0.34 & $(.01)$ & 0.85 & $(.01)$ & 0.51 & $(.01)$ & 1.10 & $(.02)$ \\
Synthetic & 0.40 & $(.01)$ & 0.91 & $(.01)$ & 0.53 & $(.01)$ & 1.09 & $(.03)$ \\
Syn.+PAGB & 0.34 & & 0.87 & & 0.52 & & 1.07 & \\
\hline
\end{tabular}

Observational data are from the compilation by Harris (1999), as dereddened according to the relations given by Reed et al. (1988). On theoretical side, we followed Scalo (1986) assuming a Salpeter exponential law down to $M_{\text {inf }}=0.3 M_{\odot}$ and a flatter function down to $0.1 M_{\odot}$. However, neither moving $M_{\text {inf }}$ up to $0.6 M_{\odot}$ or down to $0.2 M_{\odot}$ (see Table 3 ) nor changing the IMF slope (see Table 4) causes significant variations of the integrated colours (see also Kurth et al. 1999).

Because of the statistic nature of the approach, one expects that theoretical results are affected by intrinsic fluctuations whose amplitude decreases when increasing the number of stars populating the synthetic cluster. To cope with such an evidence, numerical simulations have been performed by populating the synthetic cluster till reaching the observed cluster $V$ magnitude at half-light radius.

For each case the random procedure has been repeated 10 times and Table 2 gives for each colour the obtained mean value together with the corresponding root mean square. Taking into account the uncertainty in M 30 observational data (Reed 1985), one can conclude that our synthetic approach is reproducing the colours of the actual cluster within observational uncertainties and theoretical statistical fluctuations. On the contrary, one finds that our predictions for 47 Tuc give fairly redder $U-B$ and $B-V$ colours, whereas both observational and theoretical data appear rather firmly established. This puzzling evidence is easily understood when recalling that Lloyd Evans (1974) discovered near the cluster center a hot luminous postasymptotic giant branch, for which Dixon et al. (1995) give a temperature of $\sim 10000 \mathrm{~K}$ and $\log g \leq 2.5$. Assuming a mass of $0.6 M_{\odot}$ one thus derives $\log L / L_{\odot} \sim 3.5$. As shown in the last row of Table 2 , adding the contribution of a similar object to the integrated cluster light, theory and observation appear in good agreement. Such an occurrence reinforces the already discussed evidence that integrated colours of even rich galactic globular like 47 Tuc are still affected by the stochastic presence of luminous stars in the fast phase of PAGB evolution.

Beyond such a rare occurrence, one is in all cases facing an uncertainty in predicted colour as due to statistical fluctuation in the population of bright stars. As already discussed in Paper I, one can generalize the numerical experiments presented in the previous section for M 30 and 47 Tuc by investigating the expected fluctuations of the predicted colours as a function of the integrated cluster $V$-magnitude. This because statistical fluctuations in the 
Table 3. The effects of changing the slope of the mass distribution of low mass stars for two assumptions of the integrated $M_{V}$ magnitude: $M_{\mathrm{inf}}$ is the lower limit of the Salpeter law $(x=1.35)$. From $M_{\mathrm{inf}}$ down to $0.1 M_{\odot}$ a flatter distribution with $x=0.2$ is assumed

\begin{tabular}{clllll}
\hline$M_{\text {inf }}\left(M_{\odot}\right)$ & $M_{V}$ & $U-B$ & $B-V$ & $V-R$ & $V-I$ \\
\hline 0.2 & $-4.17 \pm 0.10$ & $0.43 \pm 0.05$ & $0.92 \pm 0.05$ & $0.54 \pm 0.03$ & $1.09 \pm 0.08$ \\
0.4 & $-4.16 \pm 0.105$ & $0.43 \pm 0.05$ & $0.91 \pm 0.05$ & $0.53 \pm 0.03$ & $1.07 \pm 0.08$ \\
0.5 & $-4.15 \pm 0.11$ & $0.43 \pm 0.05$ & $0.91 \pm 0.05$ & $0.53 \pm 0.03$ & $1.07 \pm 0.08$ \\
0.6 & $-4.13 \pm 0.11$ & $0.43 \pm 0.05$ & $0.91 \pm 0.05$ & $0.53 \pm 0.035$ & $1.06 \pm 0.08$ \\
\hline 0.2 & $-7.38 \pm 0.03$ & $0.43 \pm 0.01$ & $0.93 \pm 0.01$ & $0.55 \pm 0.01$ & $1.12 \pm 0.02$ \\
0.4 & $-7.37 \pm 0.03$ & $0.43 \pm 0.02$ & $0.92 \pm 0.02$ & $0.54 \pm 0.01$ & $1.10 \pm 0.02$ \\
0.5 & $-7.36 \pm 0.03$ & $0.43 \pm 0.01$ & $0.92 \pm 0.01$ & $0.54 \pm 0.01$ & $1.10 \pm 0.02$ \\
0.6 & $-7.35 \pm 0.03$ & $0.43 \pm 0.01$ & $0.92 \pm 0.01$ & $0.54 \pm 0.01$ & $1.10 \pm 0.02$ \\
\hline
\end{tabular}

Table 4. The effects of changing the slope of the IMF for $M>M_{\text {inf }}=0.3 M_{\odot}$ : a flatter $(x=0.35)$ and steeper $(x=2.35)$ IMF than the Salpeter law are assumed

\begin{tabular}{clllll}
\hline$x$ & $M_{V}$ & $U-B$ & $B-V$ & $V-R$ & $V-I$ \\
\hline 0.35 & $-7.52 \pm 0.04$ & $0.43 \pm 0.015$ & $0.92 \pm 0.02$ & $0.54 \pm 0.015$ & $1.10 \pm 0.04$ \\
1.35 & $-7.35 \pm 0.03$ & $0.43 \pm 0.01$ & $0.92 \pm 0.01$ & $0.54 \pm 0.01$ & $1.10 \pm 0.02$ \\
2.35 & $-7.52 \pm 0.15$ & $0.42 \pm 0.015$ & $0.91 \pm 0.02$ & $0.54 \pm 0.01$ & $1.10 \pm 0.04$ \\
\hline
\end{tabular}
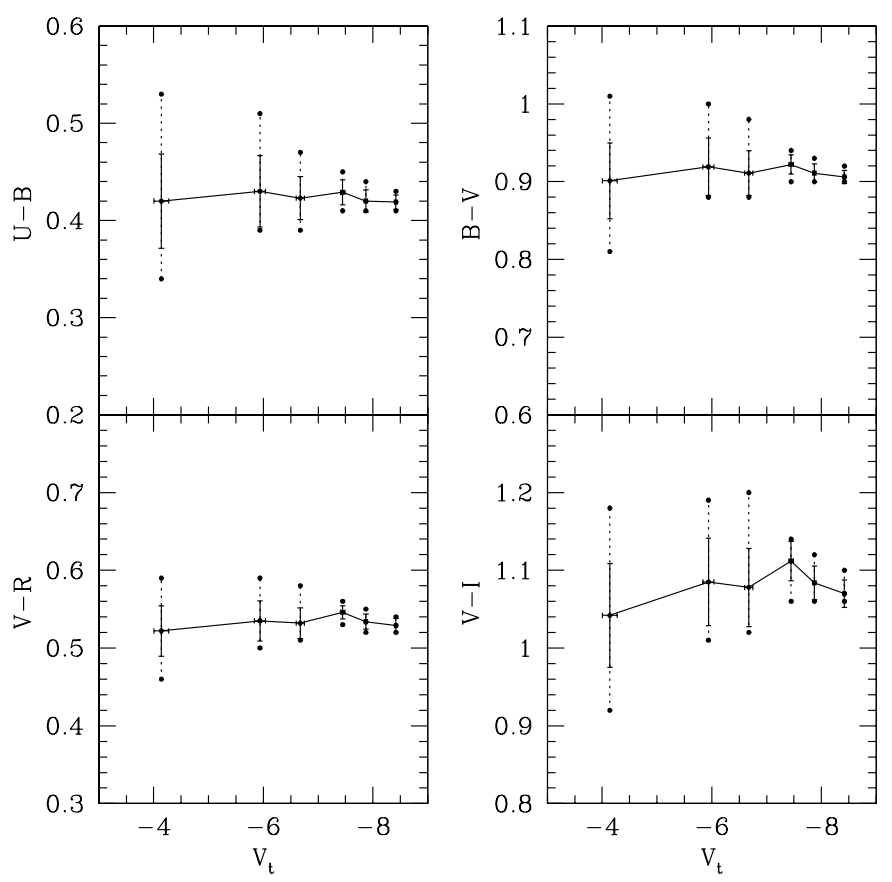

Fig. 5. Predicted stochastic fluctuations of integrated colours for a population of $15 \mathrm{Gyr}$ and $Z=0.006$ as a function of the integrated cluster visual magnitude, error bars refer to one $\sigma$. Filled circles show the maximum/minimum values obtained for each colour

population of bright stars depend on their number, which - in turn - governs the total cluster luminosity. Figure 5 gives a quantitative estimate of such an uncertainty, showing the range of colours predicted by our computations over ten different random simulations for any given assumption about the cluster luminosity.
As expected, one finds that the statistically uncertainty decreases when the integrated $V$-magnitude of the cluster increases, being reduced to few hundreds of magnitude only for cluster luminosities larger than $M_{V}^{\text {tot }} \sim-9 \mathrm{mag}$, i.e. for a magnitude reached by few very rich galactic globulars, like 47 Tuc $\left(M_{V}^{\text {tot }}=-9.42\right)$ or NGC 2419 (-9.58), NGC 6388 (-9.82), NGC 6402 $(-9.02)$, etc. On the contrary, data in Fig. 5 shows that the colours of the large majority of galactic globulars can be sensitively affected by intrinsic uncertainties. Note the increased uncertainty in $V-I$ colours, due to the stochastic contribution of cool luminous AGB stars.

Before closing this discussion it is worth mentioning that the dynamical evolution of a cluster could affect the distribution of stars and then influence the integrated colours in the region of our interest $\left(r<r^{0.5}\right)$. As a simple insight on the problem, we address the attention on two major effects of the long-term evolution driven by twobody relaxation: i) a re-distribution of stars, due to the mass segregation (King et al. 1995), and ii) a process of star loss. Both the effects cause a flattening of the IMF as the dynamical evolution of the cluster proceeds, since the evaporation rate due to two-body relaxation is larger for low mass than for high mass stars as a consequence of mass segregation (Spitzer 1987; Bolte 1989; Vesperini $\&$ Heggie 1997). Concerning our model (at $r<r^{0.5}$ ) the displacement of lower masses from central region to outer parts of globulars and/or the evaporation of stars can be simulated by subtracting low mass stars. This means that the influence of these mechanisms on integrated colours can be evaluated by analyzing the models computed for different assumptions about the slope of the IMF at low masses (Table 3 ). The result is that in poorest clusters $\left(M_{V} \sim-4.1 \mathrm{mag}\right)$ as in richest ones $\left(M_{V} \sim-7.3 \mathrm{mag}\right)$ 


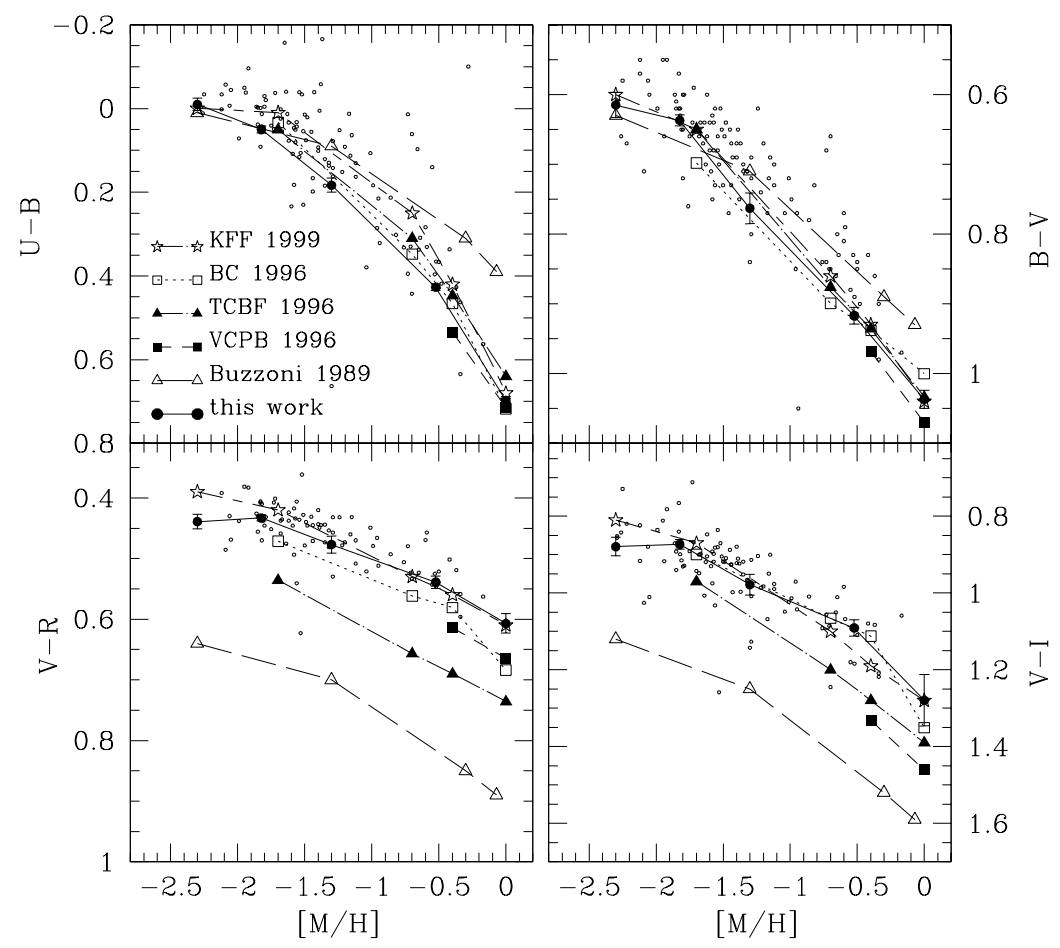

Fig. 6. Observed integrated colours of galactic globular clusters (Harris 1999). Theoretical predictions by this work and other authors are also shown (BC 1996 refers to Bruzual and Charlot models see Leitherer et al. 1996)

Table 5. Synthetic integrated colours computed assuming a constant number of He-burning stars $\left(N_{\mathrm{HB}}=300\right)$, a Salpeter IMF and $t=15$ Gyr for three different values of the Reimers parameter $\eta\left(\sigma_{\mathrm{M}}=0.04\right)$

\begin{tabular}{clllll}
\hline$Z$ & $M_{V}$ & $U-B$ & $B-V$ & $V-R$ & $V-I$ \\
\hline \multicolumn{5}{c}{$\eta=0.2$} \\
\hline 0.0001 & $-7.92 \pm 0.04$ & $0.028 \pm 0.015$ & $0.65 \pm 0.02$ & $0.46 \pm 0.01$ & $0.91 \pm 0.02$ \\
0.0003 & $-7.86 \pm 0.06$ & $0.07 \pm 0.02$ & $0.71 \pm 0.03$ & $0.47 \pm 0.02$ & $0.94 \pm 0.04$ \\
0.001 & $-7.81 \pm 0.05$ & $0.19 \pm 0.02$ & $0.81 \pm 0.02$ & $0.50 \pm 0.01$ & $1.01 \pm 0.02$ \\
0.006 & $-7.37 \pm 0.04$ & $0.45 \pm 0.01$ & $0.94 \pm 0.02$ & $0.56 \pm 1.02$ & $1.16 \pm 0.05$ \\
0.02 & $-7.05 \pm 0.03$ & $0.70 \pm 0.01$ & $1.04 \pm 0.01$ & $0.61 \pm 0.02$ & $1.32 \pm 0.07$ \\
\hline \multicolumn{5}{c}{$\eta=0.4$} \\
\hline 0.0001 & $-7.76 \pm 0.05$ & $-0.01 \pm 0.015$ & $0.62 \pm 0.02$ & $0.44 \pm 0.01$ & $0.88 \pm 0.02$ \\
0.0003 & $-7.74 \pm 0.03$ & $0.05 \pm 0.01$ & $0.65 \pm 0.01$ & $0.43 \pm 0.005$ & $0.87 \pm 0.01$ \\
0.001 & $-7.78 \pm 0.05$ & $0.18 \pm 0.02$ & $0.76 \pm 0.02$ & $0.48 \pm 0.01$ & $0.98 \pm 0.03$ \\
0.006 & $-7.35 \pm 0.03$ & $0.43 \pm 0.01$ & $0.92 \pm 0.01$ & $0.54 \pm 0.01$ & $1.10 \pm 0.02$ \\
0.02 & $-7.24 \pm 0.02$ & $0.70 \pm 0.01$ & $1.04 \pm 0.01$ & $0.61 \pm 0.02$ & $1.28 \pm 0.07$ \\
\hline \multicolumn{5}{c}{$\eta=0.6$} \\
\hline 0.0001 & $-7.64 \pm 0.06$ & $-0.06 \pm 0.02$ & $0.61 \pm 0.02$ & $0.43 \pm 0.01$ & $0.865 \pm 0.025$ \\
0.0003 & $-7.56 \pm 0.04$ & $0.003 \pm 0.02$ & $0.63 \pm 0.02$ & $0.43 \pm 0.01$ & $0.87 \pm 0.02$ \\
0.001 & $-7.52 \pm 0.09$ & $0.10 \pm 0.02$ & $0.70 \pm 0.02$ & $0.45 \pm 0.01$ & $0.92 \pm 0.02$ \\
0.006 & $-7.32 \pm 0.05$ & $0.39 \pm 0.02$ & $0.87 \pm 0.02$ & $0.52 \pm 0.01$ & $1.06 \pm 0.03$ \\
0.02 & $-7.07 \pm 0.02$ & $0.69 \pm 0.01$ & $1.03 \pm 0.01$ & $0.60 \pm 0.01$ & $1.27 \pm 0.05$ \\
\hline
\end{tabular}

the reduced number of stars with mass lower than $0.6 M_{\odot}$ does not affect the integrated colours. This is easy to understand if one considers that the contribution of stars with $M \lesssim 0.6 M_{\odot}$ to the total $V$ light is only of the order of $2-3 \%$ in the extreme case $M_{\text {inf }}=0.2 M_{\odot}$. An additional loss of stars due to dynamics can be caused by the disc shocking, which however needs a sizeable mass segregation to produce a differential escape of stars with different masses. For our purpose this case can again be re-conducted to a variation of the IMF at low masses, with similar results. Of course, a detailed treatment of the dynamical processes is more appropriate to study the problem, since peculiar spatial distribution of stars and/or star losses may affect the integrated colours and their statistical fluctuations, but it is beyond the purpose of this paper. 
Table 6. Synthetic integrated colours as in Table 3 but for different ages $\left(\eta=0.4\right.$ and $\left.\sigma_{\mathrm{M}}=0.04\right)$

\begin{tabular}{clllll}
\hline$Z$ & $M_{V}$ & $U-B$ & \multicolumn{1}{c}{$B-V$} & $V-R$ & $V-I$ \\
\hline \multicolumn{5}{c}{ age $=15 \mathrm{Gyr}$} \\
\hline 0.0001 & $-7.76 \pm 0.05$ & $-0.01 \pm 0.015$ & $0.62 \pm 0.02$ & $0.44 \pm 0.01$ & $0.88 \pm 0.02$ \\
0.0003 & $-7.74 \pm 0.03$ & $0.05 \pm 0.01$ & $0.65 \pm 0.01$ & $0.43 \pm 0.005$ & $0.87 \pm 0.01$ \\
0.001 & $-7.78 \pm 0.05$ & $0.18 \pm 0.02$ & $0.76 \pm 0.02$ & $0.48 \pm 0.01$ & $0.98 \pm 0.03$ \\
0.006 & $-7.35 \pm 0.03$ & $0.43 \pm 0.01$ & $0.92 \pm 0.01$ & $0.54 \pm 0.01$ & $1.10 \pm 0.02$ \\
0.02 & $-7.24 \pm 0.02$ & $0.70 \pm 0.01$ & $1.04 \pm 0.01$ & $0.61 \pm 0.02$ & $1.28 \pm 0.07$ \\
\hline \multicolumn{7}{c}{ age $=12 \mathrm{Gyr}$} \\
\hline 0.0001 & $-7.84 \pm 0.05$ & $0.002 \pm 0.02$ & $0.60 \pm 0.02$ & $0.43 \pm 0.02$ & $0.86 \pm 0.03$ \\
0.0003 & $-7.73 \pm 0.05$ & $0.04 \pm 0.01$ & $0.66 \pm 0.02$ & $0.44 \pm 0.01$ & $0.88 \pm 0.02$ \\
0.001 & $-7.78 \pm 0.04$ & $0.15 \pm 0.015$ & $0.77 \pm 0.02$ & $0.48 \pm 0.01$ & $0.97 \pm 0.02$ \\
0.006 & $-7.56 \pm 0.04$ & $0.39 \pm 0.015$ & $0.90 \pm 0.015$ & $0.53 \pm 0.01$ & $1.09 \pm 0.02$ \\
0.02 & $-7.23 \pm 0.01$ & $0.64 \pm 0.01$ & $1.01 \pm 0.01$ & $0.60 \pm 0.01$ & $1.28 \pm 0.05$ \\
\hline \multicolumn{7}{c}{ age $=10 \mathrm{Gyr}$} \\
\hline 0.0001 & $-7.91 \pm 0.04$ & $-0.002 \pm 0.015$ & $0.61 \pm 0.03$ & $0.43 \pm 0.02$ & $0.86 \pm 0.04$ \\
0.0003 & $-7.98 \pm 0.03$ & $0.03 \pm 0.01$ & $0.66 \pm 0.02$ & $0.44 \pm 0.01$ & $0.88 \pm 0.02$ \\
0.001 & $-7.73 \pm 0.03$ & $0.15 \pm 0.01$ & $0.76 \pm 0.02$ & $0.47 \pm 0.01$ & $0.96 \pm 0.02$ \\
0.006 & $-7.44 \pm 0.03$ & $0.36 \pm 0.01$ & $0.88 \pm 0.015$ & $0.53 \pm 0.01$ & $1.08 \pm 0.04$ \\
0.02 & $-7.17 \pm 0.03$ & $0.60 \pm 0.01$ & $0.99 \pm 0.02$ & $0.58 \pm 0.02$ & $1.26 \pm 0.07$ \\
\hline \multicolumn{7}{c}{ age $=8 \mathrm{Gyr}$} \\
\hline 0.0001 & $-8.13 \pm 0.03$ & $-0.005 \pm 0.01$ & $0.62 \pm 0.01$ & $0.435 \pm 0.01$ & $0.87 \pm 0.02$ \\
0.0003 & $-7.97 \pm 0.03$ & $0.02 \pm 0.01$ & $0.66 \pm 0.01$ & $0.43 \pm 0.01$ & $0.87 \pm 0.02$ \\
0.001 & $-7.58 \pm 0.04$ & $0.13 \pm 0.015$ & $0.74 \pm 0.02$ & $0.465 \pm 0.01$ & $0.96 \pm 0.025$ \\
0.006 & $-7.49 \pm 0.03$ & $0.32 \pm 0.01$ & $0.86 \pm 0.01$ & $0.51 \pm 0.01$ & $1.06 \pm 0.03$ \\
\hline \multicolumn{7}{c}{}
\end{tabular}

Bearing this in mind, Fig. 6 compares current predictions as obtained for a cluster age of 15 Gyr with observational data for 147 galactic globulars from Harris (1999). The rather satisfactory agreement makes us confident about the reliability of the simulations we will discuss further on. The same Fig. 6 compares present with other theoretical predictions as given in the current literature. We regard the good agreement with the results recently presented by Kurt et al. (1999) on the basis of Padua isochrones (Fagotto 1994 and references therein) as an evidence that the still existing difference in the evolutionary results (see, e.g., Castellani et al. 2000 for a discussion on that matter) are of minor relevance as far as integrated cluster colours is concerned.

Figure 7 shows that present results appear in reasonable agreement with the two colour $(U-B),(B-V)$ diagram for globulars in both the Galaxy and in the Large Magellanic Clouds without the intervention of the artificial shift invoked in Girardi et al. (1995). The same figure shows that the distribution of Large Magellanic Clouds clusters appears well fitted by predictions for young clusters already presented in Paper I.

The "solidity" of the previous results vis-a-vis the assumptions about the efficiency of mass loss has been investigated by repeating the previous computations but with the two new assumptions $\eta=0.2$ or 0.6 . The results, as presented in Table 5 , show that the case $\eta=0.2$ runs against observations (Fig. 8), whereas the increase of $\eta$ from 0.4 to 0.6 has little effect on the predicted

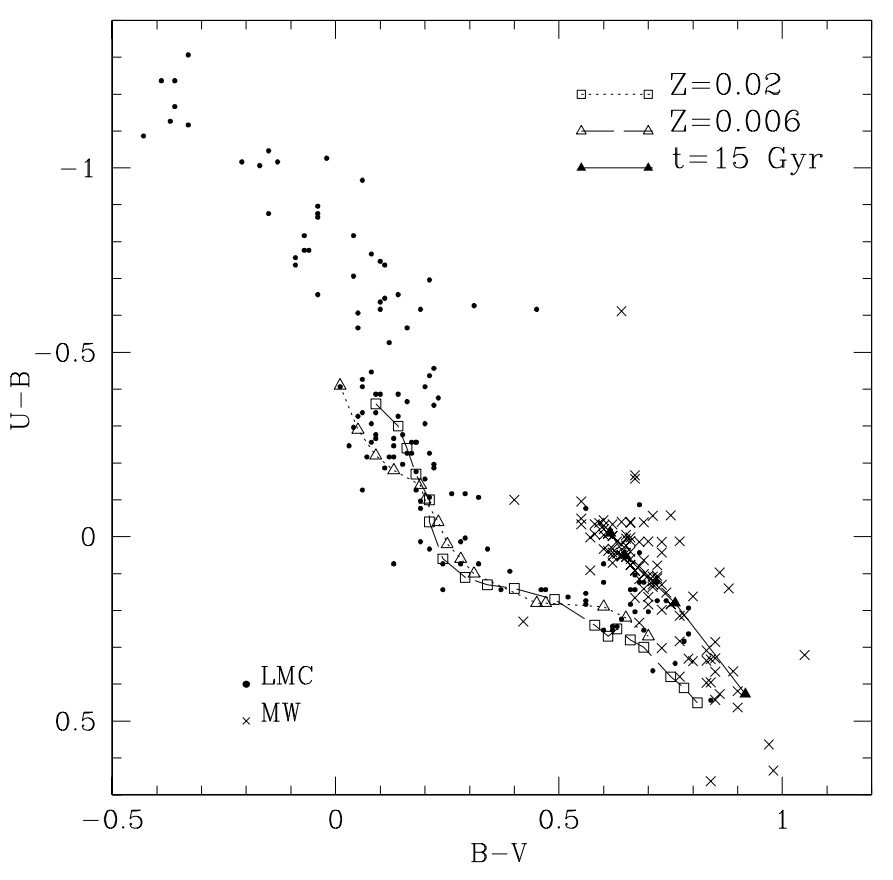

Fig. 7. Two colour diagram of observed integrated colours of LMC (filled circles) and galactic stellar clusters (diagonal crosses). Theoretical integrated colours by this work models with a fixed age $t=15 \mathrm{Gyr}$ and different chemical composition (solid line) and by Paper I models of ages ranging from $30 \mathrm{Myr}$ to 5 Gyr for two different metallicities $Z=0.02$ (dashed line) and $Z=0.006$ (dotted line) are also plotted 


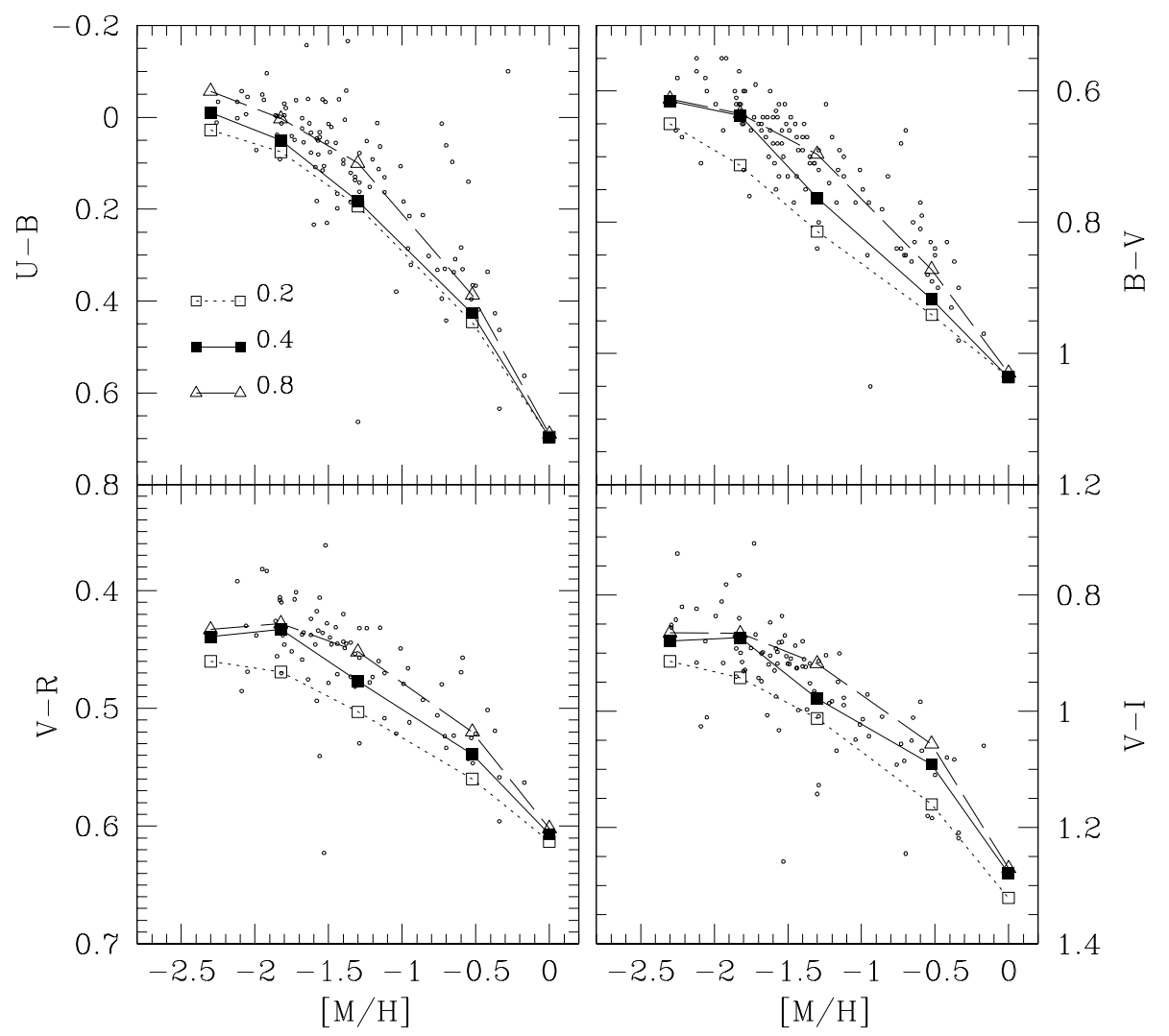

Fig. 8. Observed integrated colours of galactic globular clusters (Harris 1999) are compared to models $(t=15$ Gyr) of with different labelled assumption on $\eta$

colours: only $U-B$ colours for metal poor clusters appear marginally affected by the increase of mass loss, as expected from the evidence that such a colour is governed by the hot HB populations which, in turn, sensitively depends on the amount of mass loss. One may notice that the inadequacy of the 0.2 case would not be revealed by the two colour diagram in Fig. 7, since data in Fig. 8 show that the location of old clusters in this diagram tends to degenerate with respect of such a parameter. Conversely, one may conclude that predictions concerning $(U-B)$ vs. $(B-V)$ colours have a peculiar solidity against uncertainties in the amount of mass loss.

Finally, Fig. 9 and Table 6 show theoretical colours for selected assumptions about the cluster age, in the range 8 to $15 \mathrm{Gyr}$. As expected, one finds that decreasing the age the clusters tend to become redder. However, the amount of such a variation is so small that, taking into account the already discussed statistical uncertainties, one concludes that integrated colours are of little use to constrain the cluster ages within the above reported limits.

\section{Conclusions}

In this paper we have presented new integrated colours as computed for simple stellar population adopting a fully homogeneous set of evolutionary computations cov- ering all the major evolutionary phases of globular cluster stars. We find that, adopting Reimers parameterization of mass loss rate, the choice $\eta=0.4$ appears in satisfactory agreement with the distribution of globular cluster stars in the CM diagram. We present theoretical predictions concerning $U-B, B-V, V-R$ and $V-I$ colours for selected assumption about the cluster metallicity in the range $Z=0.0001$ to 0.02 and for ages between 8 and 15 Gyr, finding that for an age $t=15$ Gyr all the predicted colours appear in good agreement with available data for both galactic and LMC old globulars.

We discuss the uncertainties of integrated colour due to statistical fluctuations in the number of luminous stars, giving for each colour the predicted uncertainty as a function of the cluster integrated $V$-magnitude. We finally show that reasonable uncertainties either in the cluster age or in the efficiency of mass loss have marginal effects on the predicted colours of such old SSPs.

Acknowledgements. We thank G. Piotto for kindly providing us with the photometric data. This work is supported by the Italian Ministry of University, Scientific Research and Technology (MURST): Cofin1998-Project: Stellar Evolution. 


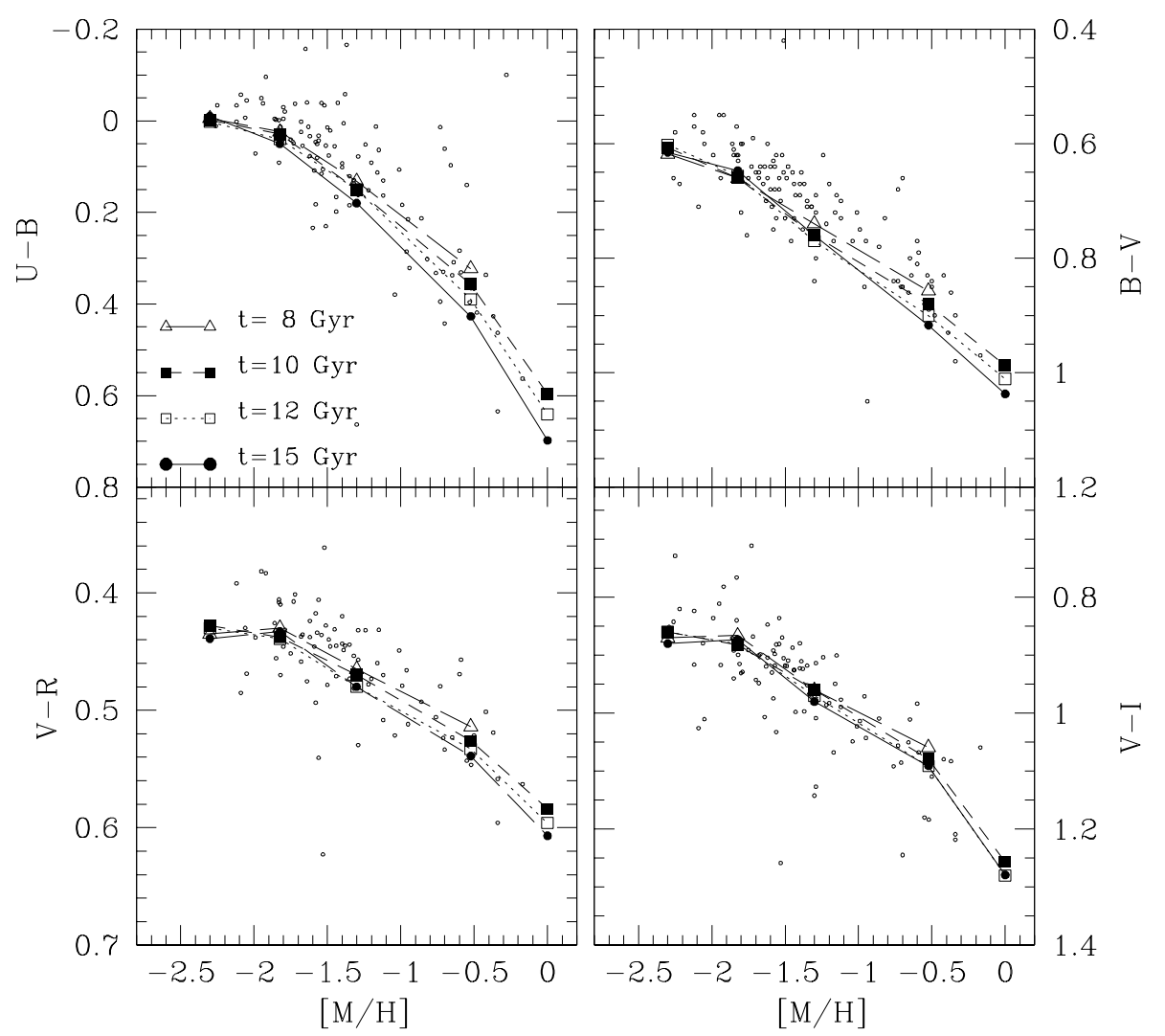

Fig. 9. Observed integrated colours of galactic globular clusters (Harris 1999) are compared to models $(\eta=0.4)$ with different labelled age

\section{References}

Blöcker T., Schönberner D., 1997, A\&A 324, 991

Bolte M., 1989, ApJ 341, 168

Brocato E., Castellani V., Raimondo G., Romaniello M., 1999a, A\&AS 136, 65 (Paper I)

Brocato E., Castellani V., Romaniello M., 1999b, A\&A 345, 499

Buzzoni A., 1989, ApJS 71, 817

Caputo F., Castellani V., Marconi M., Ripepi V., 1999, MNRAS 306, 815

Cassisi S., Castellani V., Ciarcelluti P., Piotto G., Zoccali M., 2000, MNRAS 315, 679

Cassisi S., Castellani V., Degl'Innocenti S., Weiss A., 1998, A\&AS 129, 267

Cassisi S., Castellani V., Degl'Innocenti S., Salaris M., Weiss A., 1999, A\&A 134, 103

Cassisi S., Castellani V., Straniero O., 1994, A\&A 282, 753

Castellani M., Castellani V., 1993, ApJ 407, 649

Castellani V., Ciacio F., Degl'Innocenti S., Fiorentini G., 1997, A\&A 322, 801

Castellani V., Chieffi S., Pulone L., 1991, ApJS 76, 911

Castellani V., Chieffi S., Straniero O., 1992a, ApJS 78, 517

Castellani V., Limongi M., Tornambè, 1992b, ApJ 389, 227

Castellani V., Degl'Innocenti S., Girardi L., Marconi M., Prada Moroni P.G., Weiss A., 2000, A\&A 354, 150

Castelli F., Gratton R.G., Kurucz R.L., 1997, A\&A 318, 841

Chaboyer B., 1995, ApJ 444, L9

Chaboyer B., Kim Y.-C., 1995, ApJ 454, 767
Covino S., Fracassini L., Pasinetti E., Malagnini M.L., Buzzoni A., 1994, A\&A 289, 775

Dixon W. Van Dyke, Davidsen A.F., Ferguson H.C., 1995, ApJ 454, L47

Fagotto F., Bressan A., Bertelli G., Chiosi C., 1994, A\&AS 105, 39

Gilliland R.L., Bono G., Edmonds P.D., et al., 1998, ApJ 507, 818

Girardi L., Chiosi C., Bertelli G., Bressan A., 1995, A\&A 298, 87

Groenewegen M.A.T., De Jong T., 1993, A\&A 267, 410

Harris W.E., 1999,

http://physun.physics.mcmaster.ca/GC//mwgc.dat

Iben I. Jr., Renzini A., 1983, ARA\&A 21, 271

Jørgensen U.G., Thejll P., 1993, A\&A 272, 255

King I.R., Sosin C., Cool A.M., 1995, ApJ 452, L33

Kurth O.M., Fritze-v. Alvensleben U., Fricke K.J., 1999, A\&AS 138, 19

Leitherer C., Alloin D., et al., 1996, PASP 108, 996

Lejeune T., Cuisinier F., Buser R., 1997, A\&AS 125, 229

Lloyd Evans T., 1974, MNRAS 167, 393300, 872

Maraston C., 1998, MNRAS 300, 872

Marigo P., Bressan A., Chiosi C., 1996, A\&A 313, 545

Piotto G., 2000 (private communication)

Poli F.M., Diploma Thesis, Univ. Roma

Pols O. R., Schroder K.-P., Hurley J. R., Tout C.A., Eggleton P.P., 1998, MNRAS 298, 525

Reed B.C., 1985, PASP 97, 120

Reed B.C., Hesser J.E., Shawl S.J., 1988, PASP 100, 545 
Renzini A., 1998, AJ 115, 245

Rood R.T., 1973, ApJ 184, 815

Salaris M., Weiss A., 1998, A\&A 335, 943

Sandquist E.L., Bolte M., Langer G.E., Hesser J.E., Mendes de Oliveira C., 1999, ApJ 518, 262

Scalo J.N., 1986, Fundam. Cosmic Phys. 11, 1

Schönberner D., 1983, ApJ 272, 708

Spitzer L., 1987, in Dynamical evolution of globular clusters. Princeton University Press

Straniero O., Chieffi A., 1991, ApJS 76, 525
Tantalo R., Chiosi C., Bressan A., Fagotto F., 1996, A\&A 311, 361

VandenBerg D.A., Bolte M., Stetson P.B., 1990, AJ 100, 445

VandenBerg D.A., Stetson P.B., Bolte M., 1996, ARA\&A 34, 461

Vazdekis A., Casuso E., Peletier R.F., Beckman J.E., 1996, ApJS 106, 307

Vesperini E., Heggie D.C., 1997, MNRAS 289, 898

Wood M.A., 1992, ApJ 386, 539 